\title{
Return times and conjugates of an antiperiodic transformation
}

\author{
STEVE ALPERN† \\ From the Department of Mathematics, London School of Economics, England
}

(Received 6 June 1980 and revised 8 April 1981)

\begin{abstract}
Denote by $G$ the group of all $\mu$-preserving bijections of a Lebesgue probability space $(X, \Sigma, \mu)$ and by $C$ the conjugacy class of an antiperiodic transformation $\sigma$ in $G$. We present several new results concerning the denseness of $C$ in $G$ with respect to various topologies. One of these asserts that given any weakly mixing transformation $\tau$ in $G$ and any $F$ with $\mu(F)<1$, there is a transformation in $C$ which agrees with $\tau$ a.e. on $F$.
\end{abstract}

\section{Introduction and statement of results}

In this paper we consider two related questions concerning an arbitrary antiperiodic automorphism $\sigma$ of a Lebesgue probability space $(X, \Sigma, \mu)$. The first question concerns when we can find a sweep-out set for $\sigma$ with a specified distribution of return times. The second asks in which ways automorphisms of $X$ can be approximated by conjugates of $\sigma$.

Denote by $G=G(X, \Sigma, \mu)$ the group of all automorphisms ( $\mu$-preserving bijections) of $(X, \Sigma, \mu)$, and by $C(\sigma)$ the class of all conjugates, $\theta^{-1} \sigma \theta, \theta \in G$, of the antiperiodic automorphism $\sigma$. The first question seeks a sweep-out set $B$ such that the relative distribution of return times to $B$ is a given probability distribution $\pi=\left(\pi_{1}, \pi_{2}, \ldots\right)$. Suppose $d>1$ divides all the $k$ for which $\pi_{k}>0$. Then for any such set $B$, and any $m$ which is not a multiple of $d$, we would have

$$
\mu\left(B \cap \sigma^{m} B\right)=0 .
$$

So such a set $B$ cannot exist in general, for example when $\sigma$ is mixing. However, if no such $d$ exists for $\pi$, then the required set $B$ can always be found (by taking $B=\bigcup_{k=1}^{\infty} P_{k, 1}$ in the following).

THEOREM 1. Let $\sigma \in G$ be antiperiodic and let $\pi=\left(\pi_{1}, \pi_{2}, \ldots\right)$ be any denumerable probability distribution such that the $k s$ with $\pi_{k}>0$ are relatively prime. Then there is a partition $\left\{P_{k, i}\right\}, k=1, \ldots, \infty, i=1, \ldots, k$ of $X$ satisfying

(i) $P_{k, i}=\sigma^{i-1}\left(P_{k, 1}\right)$; and

(ii) $\mu\left(\bigcup_{i=1}^{k} P_{k, i}\right)=\pi_{k}$

† Address for correspondence: Dr Steve Alpern, Department of Mathematics, London School of Economics, Houghton Street, London, England. 
Theorem 1 generalizes corollary 2 of [1] which is restricted to finite dimensional distributions $\pi=\left(\pi_{1}, \ldots, \pi_{n}\right)$, and also Rohlin's lemma which is covered by $\pi_{1}=\varepsilon$ and $\pi_{n}=1-\varepsilon$. Basically, theorem 1 says that an antiperiodic automorphism can be represented by stacks $\left(\bigcup_{i=1}^{k} P_{k, i}\right)$ of given heights $(k)$ and given measures $\left(\pi_{k}\right)$ as long as the heights are relatively prime. The proof of theorem 1 incorporates a substantial simplification for which I wish to thank the referee.

We turn now to the second question: the approximation of an automorphism $\tau \in G$ by a conjugate $\hat{\sigma}$ of $\sigma(\hat{\sigma} \in C(\sigma))$. We list below four types of approximation, which we shall then discuss in turn.

Approximation problems. Let $\tau, \sigma \in G$ with $\sigma$ antiperiodic, $\varepsilon>0$ and $F, A_{m} \in \Sigma$, $m=1, \ldots, M$ be given. We seek a $\hat{\sigma} \in C(\sigma)$ satisfying:

(P1) $\mu\left(\hat{\sigma}\left(A_{m}\right) \Delta \tau\left(A_{m}\right)\right) \leq \varepsilon, \quad m=1, \ldots, M$;

(P2) $\mu\left(\hat{\sigma}\left(A_{m}\right) \triangle \tau\left(A_{m}\right)\right)=0, \quad m=1, \ldots, M$;

(P3) $\mu\{x \in X: \hat{\sigma}(x) \neq \tau(x)\} \leq \varepsilon$;

(P4) $\mu\{x \in F: \hat{\sigma}(x) \neq \tau(x)\}=0$.

The problem P1 was first studied by Halmos [6] and [7], who showed that P1 can be solved without any restrictions on $\tau$ or the $A_{m}$. That is, $C(\sigma)$ is dense in $G$ in the coarse, or weak topology [8, p. 77].

We next consider problem P2. First observe that, if $\hat{\sigma}$ satisfies $\mathrm{P} 2$, then $\hat{\sigma}(A)=$ $\tau(A)$ (equality is always up to sets of measure 0 ) for all $A$ belonging to the subalgebra $\mathscr{A}$ of $\Sigma$ generated by $A_{1}, \ldots, A_{M}$. Now suppose that the set map $\tau / \mathscr{A}$ has a non-trivial periodic point, that is, an $A \in \mathscr{A}-\{\varnothing, x\}$ with $\tau^{i}(A) \in \mathscr{A}$ for $i=1, \ldots, k$, and $\tau^{k}(A)=A$. Then for any $\hat{\sigma}$ satisfying P2 we have $\hat{\sigma}^{k}(A)=\tau^{k}(A)=$ $A$, so that $\hat{\sigma}$, and hence $\sigma$, could not be mixing. Consequently, P2 cannot be solved in general. However, if we exclude the above situation by hypothesis, then P2 can always be solved.

TheOREM 2. Let $\tau, \sigma \in G$ with $\sigma$ antiperiodic. Let $\mathscr{A}$ be a finite subalgebra of $\Sigma$ such that $\tau / \mathscr{A}$ has no non-trivial periodic point. Then there is a $\hat{\sigma} \in C(\sigma)$ such that $\hat{\sigma}(A)=$ $\tau(\boldsymbol{A})$ for all $\boldsymbol{A} \in \mathscr{A}$.

THEOREM 3. Furthermore. (continuing from above) let $\rho$ be any totally bounded metric on $X$ such that $\mu$ is positive on open sets. Let $D$ denote the union of all atoms of $\mathscr{A}$ whose image under $\tau$ is connected. Then, given any $\varepsilon>0$, there is a $\hat{\sigma} \in C(\sigma)$ such that $\hat{\sigma}(A)=\tau(A)$ for all $A \in \mathscr{A}$ and $\rho(\hat{\sigma}(x), \tau(x))<\varepsilon$ for (a.e.) $x$ in $D$.

We note that theorem 3 is used in [3] to prove the existence of an ergodic non-stable Lebesgue measure preserving homeomorphism of $\mathbb{R}^{4}$, conditional on the existence of a non-ergodic one.

We briefly discuss problem P3 by observing that it can be solved when $\tau$ is antiperiodic, by applying Rohlin's lemma to both $\tau$ and $\sigma$. That is, $C(\sigma)$ is dense in the antiperiodic transformations with respect to the uniform topology [5, p. 112].

Finally, we discuss the strongest type of approximation, P4. First, it is obvious that we must require $\mu(F)<1$, for otherwise P4 implies $\tau$ is conjugate to $\sigma$. Next, 
the same argument used in the discussion of $\mathrm{P} 2$ shows that we must rule out the possibility that $\tau / \mathscr{A}$ has a non-trivial periodic point, where $\mathscr{A}$ is here the $\sigma$-algebra generated by measurable subsets of $F$. This possibility is excluded by either assumption of the following result, which is proved by using theorem 1.

THEOREM 4. Let $\tau, \sigma \in G$, with $\sigma$ antiperiodic, and $F \in \Sigma$ with $\mu(F)<1$ be given. Assume either

(1) $\tau$ is ergodic and $\mu(F \cup \tau F)<1$, or

(2) $\tau$ is weakly mixing.

Then there is $a \hat{\sigma} \in C(\sigma)$ such that $\hat{\sigma}(x)=\tau(x)$ for (a.e.) $x$ in $F$.

Part (1) of theorem 4 is similar to a recent result of Choksi and Kakutani for $G(\bar{X}, \bar{\Sigma}, \bar{\mu})$, where $(\bar{X}, \bar{\Sigma}, \bar{\mu})$ is an infinite $\sigma$-finite Lebesgue measure space. They demonstrated [4, theorem 6] that when $\tau, \sigma \in G(\bar{X}, \bar{\Sigma}, \bar{\mu})$, with $\tau$ ergodic, $\sigma$ antiperiodic, and $F \in \bar{\Sigma}$ with $\mu(F)<\infty$ are given, there is a $\hat{\sigma} \in C(\sigma)$ with $\sigma(x)=\tau(x)$ for a.e. $x$ in $F$. This can be expressed by saying that $C(\sigma)$ is dense in the ergodic automorphisms with respect to the 'strong topology' defined (in [2]) by basic neighbourhoods consisting of all automorphisms (of $(\bar{X}, \bar{\Sigma}, \bar{\mu})$ ) agreeing with a given one on a given set of finite measure. Theorem 4 can be similarly expressed by saying that $C(\sigma)$ is dense in the weakly mixing automorphisms with respect to the 'compact-equal' topology on $G$ defined as follows. Identify $(X, \Sigma, \mu)$ with Lebesgue measure on the open unit interval. A basis is then given by sets of all automorphisms agreeing with a given one on a given compact set. This topology is finer than the uniform topology. For applications to the study of measure preserving homeomorphisms, $(X, \Sigma, \mu)$ can be identified with other non-compact spaces.

\section{Proof of theorem 1}

Our proof of theorem 1 will involve some special notation, for which we fix the $\sigma$. and $\pi$ of the theorem. For $j \geq 1$, let

$$
\begin{gathered}
S^{j} \equiv\left\{k \leq j: \pi_{k}>0\right\}, \\
s_{i}=\sum_{k \in S^{j}} \pi_{k},
\end{gathered}
$$

and

$$
\pi^{i}=\left(\pi_{1}^{i}, \pi_{2}^{j}, \ldots, \pi_{j}^{j}, 0,0, \ldots\right)
$$

where $\pi_{k}^{j}=\pi_{k} / s_{i}$, for $k \leq j$. According to assumption on $\pi$, there is a $j_{0}$ such that $S^{j_{0}}$ is relatively prime. Consequently, all integers greater than or equal to some fixed integer $L$ may be represented as positive integer linear combinations of $S^{j_{0}}$.

A $\pi$-partition of $X$ is a measurable partition $R=\left\{R_{k, i}\right\}, k=1, \ldots, \infty, i=1, \ldots, k$ such that

$$
R_{k, i}=\sigma^{i-1}\left(R_{k, 1}\right), \text { and } \mu\left(R_{k, 1}\right)=0
$$

whenever $\pi_{k}=0$. Define

$$
R_{k}=\bigcup_{i=1}^{k} R_{k, i}
$$


and

$$
d(R)=\left(\mu\left(R_{1}\right), \mu\left(R_{2}\right), \ldots\right) .
$$

In this notation, theorem 1 asserts the existence of a $\pi$-partition $P$ with $d(P)=\pi$.

Our proof will obtain $P$ as the limit of $\pi$-partitions $P^{j}, j \geq j_{0}$, with respect to the partition metric

$$
\|R-Q\|=\mu\{x: x \text { has different } R \text { and } Q \text { labels }\}
$$

on the (complete) space of $\pi$-partitions. Each $P^{j}$ will be a $\pi$-partition 'of type $j$ ' by which we mean that $\mu\left(P_{k}^{j}\right)=0$ for $k>j$.

To ensure that $d(P)=\pi$ we use the 'sum' metric on $l^{\infty}$ and observe that

$$
|d(R)-d(Q)|=\sum_{k=1}^{\infty}\left|\mu\left(R_{k}\right)-\mu\left(Q_{k}\right)\right| \leq 2\|R-Q\| .
$$

So we would like to choose $P^{j}$ with $\left|d\left(P^{i}\right)-\pi\right|$ very small. But unfortunately, since $P^{j}$ is of type $j,\left|d\left(P^{j}\right)-\pi\right|$ is bounded away from 0 . So instead we choose $P^{i}$ with $\left|d\left(P^{i}\right)-\pi^{j}\right|$ small, or equivalently, with $\Delta_{j}\left(P^{i}\right)$ small, where

$$
\Delta_{i}(R)=\max _{k \in S_{i}}\left(1-\pi_{k}^{j} / \mu\left(R_{k}\right)\right) \text {. }
$$

The construction of the $P^{i}$ will be based on the following two lemmas.

LEMMA 1. For any positive integer $n$ there is a sweep-out set $B=B_{n}$ whose return times are not less than $n$. That is, there is a measurable subset $B$ of $X$ satisfying:

(i) the sets $B, T B, \ldots, T^{n-1} B$ are disjoint; and

(ii) $\bigcup_{l=1}^{\infty} T^{l} B=X$

Proof. This result is, of course, a special case of the finite dimensional version of theorem 1 [1, corollary 2] which gives us (for example) a sweep-out set whose only return times are $n$ and $n+1$. However, the lemma as formulated may be established directly by observing that any set which is maximal with respect to (i) must necessarily also satisfy (ii) [8, pp. 70-72].

Lemma 2. Let $j \geq j_{0}$ and $\varepsilon>0$ be given. Then to every $\pi$-partition $R$ of type $j$ there corresponds a $\pi$-partition $Q$ of type $j$ satisfying $\left|d(Q)-\pi^{j}\right|<\varepsilon$ and $\|Q-R\| \leq \Delta_{i}(R)$. Proof. Let $B=B_{n}$ be the set given by lemma 1 for some large $n$ to be specified later. Partition $B$ into sets $B^{l}, l=1,2, \ldots$ so that, if $x, y \in B^{l}$, then $x$ and $y$ have the same return time $n^{l}$ to $B$, and $\sigma^{m}(x)$ and $\sigma^{m}(y)$ belong to the same element of $R$ for $m=0, \ldots, n^{l}-1$. Next partition each $B^{l}$ into sets $B_{0}^{l}$ and $B_{k}^{l}, k \in S^{j}$, so that

$$
\mu\left(B_{0}^{l} / B^{l}\right)=\alpha_{k} \quad \text { and } \mu\left(B_{k}^{l} / B^{l}\right)=\beta_{k},
$$

where $\alpha=1-\Delta_{j}(R)$ and $\beta_{k}=\pi_{k}^{j}-\alpha \mu\left(R_{k}\right) \geq 0$. Let $C_{k}^{l}$ be the 'column' based on $B_{k}^{l}$, that is

and let $D_{k}=\bigcup_{l} C_{k}^{l}$. Observe that

$$
C_{k}^{l}=\bigcup_{m=0}^{n^{l}-1} \sigma^{m}\left(B_{k}^{l}\right),
$$

$$
\mu\left(D_{0}\right)=\alpha=1-\Delta_{j}(R), \quad \mu\left(D_{k}\right)=\beta_{k},
$$


and that

$$
\mu\left(R_{k} \cap D_{0}\right)=\alpha \mu\left(R_{k}\right)=\pi_{k}^{j}-\beta_{k}
$$

We now define $Q$ on $D_{0}$ to be the same as $R$. Regardless of how we subsequently define $Q$ on the complement $\sim D_{0}$ of $D_{0}$ we shall have

$$
\|Q-R\| \leq \mu\left(\sim D_{0}\right)=1-\alpha=\Delta_{j}(R) .
$$

If we could define $Q$ on $\sim D_{0}$ so that $\mu\left(Q_{k} / D_{k}\right)=1$, we would have

$$
\mu\left(Q_{k}\right) \geq \mu\left(R_{k} \cap D_{0}\right)+\mu\left(Q_{k} / D_{k}\right) \mu\left(D_{k}\right)=\alpha \mu\left(R_{k}\right)+\beta_{k}=\pi_{k}^{j},
$$

and consequently $d(Q)=\pi^{j}$. By defining $Q$ on $D_{k}$ so that $\mu\left(Q_{k} / D_{k}\right)$ is nearly 1 , we shall ensure that

$$
\left|d(Q)-\pi^{i}\right|<\varepsilon
$$

We define $Q$ on $D_{k}$ by specifying it on each column

$$
C_{k}^{l}=\bigcup_{m=0}^{n^{l}-1} \sigma^{m}\left(B_{k}^{l}\right)
$$

as follows. For simplicity take $B_{k}^{l}=E$ and $n^{l}=N$. Suppose $E \subset R_{\left(k_{1}, i_{1}\right)}$ and $\sigma^{N-1}(E) \in R_{\left(k_{2}, i_{2}\right)}$. We assign $N Q$-labels to the sets $E, \sigma E, \sigma^{2} E, \ldots, \sigma^{N-1} E$ by beginning and ending with $R$-labels:

$$
\left(k_{1}, i_{1}\right),\left(k_{1}, i_{1}+1\right), \ldots,\left(k_{1}, k_{1}\right),-,-, \ldots,-,-,\left(k_{2}, 1\right),\left(k_{2}, 2\right), \ldots,\left(k_{2}, i_{2}\right) .
$$

We then fill in successive blocks of the form

$$
(k, 1),(k, 2), \ldots,(k, k),
$$

beginning immediately after $\left(k_{1}, k_{1}\right)$, until there are $T$ blanks remaining between the final $(k, k)$ and the label $\left(k_{2}, 1\right)$, where $T$ satisfies $L \leq T \leq L+k$. Since $T \geq L$, the definition of $L$ guarantees that these blanks may be filled in with blocks of the form

$$
\left(k^{\prime}, 1\right),\left(k^{\prime}, 2\right), \ldots,\left(k^{\prime}, k^{\prime}\right),
$$

where $k^{\prime} \in S^{j_{0}}$. This procedure ensures that $Q$ is a $\pi$-partition. Furthermore, of the $N$ labels in this sequence, all but at most

$$
\left(k_{1}-i_{1}+1\right)+T+k_{2} \leq k+(T+k)+k=T+3 k \leq T+3 j
$$

are in $Q_{k}$ (first coordinate $k$ ). Thus

$$
\mu\left(Q_{k} / C_{k}^{l}\right) \geq 1-(T+3 j) / n^{l} \geq 1-(T+3 j) / n,
$$

and consequently

$$
\mu\left(Q_{k} / D_{k}\right) \geq 1-(T+3 j) / n \geq 1-\frac{1}{2} \varepsilon,
$$

if we take $n>\frac{1}{2} \varepsilon(T+3 j)$. Finally, we calculate

$$
\pi_{k}^{j}-\mu\left(Q_{k}\right) \leq \frac{1}{2} \varepsilon \beta_{k}
$$

for $k \in S^{j}$ so that

$$
\left|\pi^{j}-d(Q)\right| \leq 2\left(\frac{1}{2} \varepsilon\right) \sum_{k \in S^{i}} \beta_{k} \leq \varepsilon
$$

as required. 
Proof of theorem 1. For $j \geq j_{0}$ choose positive numbers $\varepsilon_{j}$ going to 0 and sufficiently small so that

$$
\left|d(Q)-\pi^{i}\right|<\varepsilon_{j} \text { implies } \Delta_{j}(Q)<2^{-(j+1)}
$$

for any $\pi$-partition $Q$. For $j \geq j_{0}$ we construct a $\pi$-partition $P^{i}$ of the type $j$ satisfying:

(i) $\left|d\left(P^{i}\right)-\pi^{i}\right|<\varepsilon_{j}$; and

(ii) $\left\|P^{j}-P^{j-1}\right\| \leq 2^{-i}+\pi_{j} / s_{j} \quad\left(j>j_{0}\right)$.

It then follows that $\left\|P^{i}-P\right\| \rightarrow 0$ for some $\pi$-partition $P$, which necessarily satisfies $d(P)=\pi$ (see remarks preceeding lemma 1 ).

The $P^{j}$ are constructed as follows. The first one, $P^{i_{0}}$, satisfying property (i), may be obtained directly from the finite version of theorem 1 [1, corollary 2$]$ - in fact with $d\left(P^{i_{0}}\right)=\pi^{j_{0}}$. However, we may make this proof self-contained by observing that the algorithm of lemma 2, used with $\alpha=0$ and $\beta_{k}=\pi_{k}^{i_{0}}$, yields the required $\pi$-partition $P^{i_{0}}$ directly. Now suppose

$$
P^{i_{0}}, \ldots, P^{i-1}
$$

have been found satisfying (i) and (ii). Since $P^{i-1}$ satisfies

$$
\left|d\left(P^{j-1}\right)-\pi^{i-1}\right|<\varepsilon_{i-1}
$$

we know by choice of $\varepsilon_{j-1}$ that

$$
\Delta_{i-1}\left(P^{i-1}\right)<2^{-j} .
$$

Now observe that any $\pi$-partition $R$ of type $j-1$ is also of type $j$ and that

$$
\Delta_{j}(R) \leq \Delta_{j-1}(R)+\pi_{j} / s_{i} .
$$

If we apply this inequality to $P^{i-1}$ we obtain

$$
\Delta_{j}\left(P^{i-1}\right) \leq 2^{-i}+\pi_{j} / s_{j}
$$

Now apply lemma 2 taking $R=P^{i-1}$ and $\varepsilon=\varepsilon_{j}$ to obtain (as $Q$ ) the partition $P^{i}$ satisfying (i) and (ii).

\section{Proof of theorem 4}

Since $\tau$ is ergodic and $\mu(F)<\mu(X)$, it follows that the $\tau$-orbit of a.e. point of $F$ eventually leaves $F$. Consequently, we can partition the set

$$
F \cup \tau F=\bigcup_{k=2}^{\infty} \bigcup_{i=1}^{k} F_{k, i}
$$

where $F_{k, i}=\tau^{i-1}\left(F_{k, 1}\right), F_{k, i} \subset F-\tau F$ for $i<k$, and $F_{k, k} \subset T F-F$. Let

$$
F_{1,1}=X-(F \cup T F)
$$

and define

$$
\pi_{k}=\mu\left(\bigcup_{i=1}^{k} F_{k, i}\right) .
$$

We claim that the $k \mathrm{~s}$ for which $\pi_{k}>0$ are relatively prime. The demonstration of this fact breaks up into two cases. In case (1), the hypothesis $\mu(F \cup T F)<\mu(X)$ ensures that $\pi_{1}>0$. Next suppose that we are in case (2), but not case (1), so that $\tau$ is weakly mixing, and $\mu(F \cup T F)=\mu(X)$. Suppose that $p$, the greatest common 
divisor of the $k$ s for which $\pi_{k}>0$, is greater than 1 . Let $D=F_{k, 1}$ for some $k$ with $\pi_{k}>0$. Then

$$
\mu(X)>\mu(D)=\pi_{k} / k>0
$$

but

$$
\mu\left(D \cap \tau^{n p+1} D\right)=0 \text { for all } n .
$$

But this contradicts the hypothesis that $\tau$ is weakly mixing, so we must have $p=1$. Thus in either case, $\pi$ satisfies the hypothesis of theorem 1 .

Let $P=\left\{P_{k, i}\right\}$ be the partition given by theorem 1 to the $\sigma$ of this theorem and the distribution $\pi$ we have just constructed. Define $\theta \in G(X, \Sigma, \mu)$ so that

$$
\theta\left(P_{k, 1}\right)=F_{k, 1} \text { and } \theta(x)=\tau^{i-1} \sigma^{1-i}(x)
$$

for $x \in P_{k, i}$, for all $k$ with $\pi_{k}>0$ and all $i$ such that $1 \leq i \leq k$. It follows from this construction that

$$
\tau(x)=\theta^{-1} \sigma \theta(x)
$$

whenever $x \in F_{k, i}$ where $i<k$. But

$$
F \subset \bigcup_{k} \bigcup_{i<k} F_{k, i}
$$

so the theorem is proved.

\section{Proofs of theorems 2 and 3}

The following discussion, through the statement of proposition 1 , is taken from [1]. Let $T=\left\{t_{i j}\right\}$ be an $n \times n$ matrix consisting of 0 s and $1 \mathrm{~s}$. We call $T$ 'aperiodic' if for some integer $N, T^{N}$ has all positive entries. $T$ induces a map $\hat{T}: \Gamma \rightarrow \Gamma$, where $\Gamma$ is the power set of $\{1, \ldots, n\}$, by $j \in \hat{T}(\gamma)$ if $t_{i j}=1$ for some $i \in \gamma \in \Gamma$. Let $\Gamma_{1}$ denote the subalgebra of $\Gamma$ given by: $\gamma \in \Gamma_{1}$ if $t_{i j}=1$ and $j \in \hat{T}(\gamma)$ imply $i \in \gamma$. We say that a probability distribution $\left(p_{1}, \ldots, p_{n}\right)$ is consistent with $T$ if it satisfies

(1) $\sum_{i \in \gamma} p_{i}=\sum_{j \in \hat{f}(\gamma)} p_{j} \quad$ for all $\gamma \in \Gamma_{1}$; and

(2) $\sum_{i \in \gamma} p_{i}<\sum_{j \in \hat{T}(\gamma)} p_{j} \quad$ for all $\gamma \in \Gamma-\Gamma_{1}$.

Proposition 1 (immediate consequence of theorem 1, [1]). Let $\left\{P_{i}\right\}_{i=1}^{n}$ be a measurable partition of $(X, \Sigma, \mu)$ whose distribution $\left(\mu P_{1}, \mu P_{2}, \ldots, \mu P_{n}\right)$ is consistent with an $n \times n$ 0-1 matrix $T$. Let $\sigma \in G$ be antiperiodic. Then there is $a \hat{\sigma} \in C(\sigma)$ such that $\mu\left(\hat{\sigma} P_{i} \cap P_{j}\right)=0$ for all $i, j$ with $t_{i, j}=0$.

Proof of theorem 2. Let $A_{l}, l=1, \ldots, L$ denote the atoms of $\mathscr{A}$. Let $\left\{P_{i j}\right\}_{i, j=1}^{n}$ be a measurable partition of $(X, \Sigma, \mu)$ which refines the partitions given by the atoms of $\mathscr{A}$ and the atoms of $\tau(\mathscr{A})$. Define an $n \times n$ 0-1 matrix $T$ by

(3) $t_{i j}= \begin{cases}1 & \text { if } P_{i} \subset A_{l} \text { and } P_{j} \subset \tau\left(A_{l}\right) \text {, some } l ; \\ 0 & \text { otherwise. }\end{cases}$

First observe that $\gamma \in \Gamma_{1}$ if and only if $\bigcup_{i \in \gamma} P_{i} \in \mathscr{A}$, and that consequently $\left(\mu P_{1}, \ldots, \mu P_{n}\right)$ is consistent with $T$. To show that $T$ is aperiodic it is sufficient to 
prove that, for any $\gamma \in \Gamma-\{\varnothing\}$, the sequence

$$
\mu(\gamma), \mu(\hat{T} \gamma), \mu\left(\hat{T}^{2} \gamma\right), \ldots
$$

is eventually 1 , where $\mu(\gamma)=\sum_{i \in \gamma} \mu P_{i \text {. }}$ (If $N$ is the longest number of steps it takes, then $T^{N}>0$.) It follows from (3) that $\mu(\gamma) \leq \mu(\hat{T} \gamma)$ with equality only for $\gamma \in \Gamma_{1}$. Consequently, the only way such a sequence can fail to reach 1 is if $\hat{T} / \Gamma_{1}$ has a non-trivial period point $\gamma_{0}$. But then $A=\bigcup_{i \in \gamma_{0}} P_{i}$ would be a non-trivial periodic point of $T / \mathscr{A}$. Since this possibility is excluded by assumption, our argument shows that $T$ is aperiodic. The automorphism $\hat{\sigma}$ given by proposition 1 now proves the theorem.

Proof of theorem 3 . This proof is very similar to the proof of theorem 2 , so we only indicate the differences. Let $A_{1}, \ldots, A_{L_{1}}$ be the atoms of $\mathscr{A}$ whose $\tau$ images are connected. Let $\left\{P_{i j}\right\}, i, j=1, \ldots, n$ additionally satisfy

$$
\rho\left(P_{i}\right)<\frac{1}{2} \varepsilon \text { and } \rho\left(\tau\left(P_{i}\right)\right)<\frac{1}{2} \varepsilon,
$$

which is possible because $X$ is totally bounded $(\rho(\quad)$ denotes diameter). Define $T$ by

$$
t_{i j}= \begin{cases}1 & \text { if } P_{i} \subset A_{l} \text { and } P_{j} \subset T\left(A_{l}\right), \text { some } l>L_{1} ; \\ 1 & \text { if } P_{i} \subset A_{l} \text { and } P_{j} \subset T\left(A_{l}\right), \text { some } l \leq L_{1} \text { and } \\ & \overline{\tau P}_{i} \cap \bar{P}_{j} \neq \varnothing, \text { where bar denotes closure; } \\ 0 & \text { otherwise. }\end{cases}
$$

The proof that $T$ is aperiodic and that $\left(\mu P_{1}, \ldots, \mu P_{n}\right)$ is consistent with $T$ is the same as that for theorem 2 except that the connectivity of $T\left(A_{l}\right), l \leq L_{1}$, is used to identify $\Gamma_{1}$ with $\mathscr{A}$. Let $\hat{\sigma}$ be the automorphism given by proposition 1 . To establish the final estimate of the theorem, assume $P_{i} \subset D$, or equivalently, $P_{i} \subset A_{\text {l, }}$, some $l \leq L_{1}$. Then

$$
\rho\left(\hat{\sigma} P_{i} \cup \tau P_{i}\right) \leq \max _{i: t_{i}=1} \rho\left(P_{j} \cup \tau P_{i}\right) \leq \rho\left(P_{j}\right)+\rho\left(\tau P_{i}\right)<\varepsilon,
$$

since $\bar{P}_{i} \cap \overline{\tau P}_{i} \neq \varnothing$. It follows that

$$
\rho(\hat{\sigma}(x), \tau(x))<\varepsilon \quad \text { for a.e. } x \text { in } D .
$$

The application (in [3]) of theorem 3 mentioned in the introduction uses only the following special case.

Corollary 1. Let $(X, \Sigma, \mu, \rho)$ be as in theorem 3. Let $A \in \Sigma$ and let $\tilde{\tau}: A \rightarrow X$ be any $\mu$-preserving injection such that $\mu(\tilde{\tau}(A) \triangle A)>0$ and $\tilde{\tau}(A)$ is connected. Then given any $\varepsilon>0$ there is a $\mu$-preserving injection $\tilde{\sigma}: A \rightarrow \tilde{\tau}(A)$ such that $\rho(\tilde{\tau}(x), \tilde{\sigma}(x))<$ $\varepsilon$ for a.e. $x$ in $A$, and $\tilde{\sigma}$ has no non-trivial invariant sets.

Proof. We may assume without loss of generality that $\mu(A \cap \tilde{\tau}(A))>0$, for otherwise simply take $\tilde{\sigma}=\tilde{\tau}$. Let $\tau \in G(X, \Sigma, \mu)$ be any extension of $\tilde{\tau}$ and let $\mathscr{A}=$ $\{\varnothing, X, A, \sim A\}$. The set $D$ of theorem 3 is either $A$ or $X$, but in any case $D \supseteq A$. Let $\sigma \in G$ be any ergodic automorphism and let $\hat{\sigma}$ be the conjugate of $\sigma$ which approximates $\tau$ in the sense of theorem 3. Then the restriction $\tilde{\sigma}$ of $\hat{\sigma}$ to $A$ has the required properties. 


\section{REFERENCES}

[1] S. Alpern. Generic properties of measure preserving homeomorphisms. In Ergodic Theory: Oberwolfach. Springer Lecture Notes in Math. no. 729, pp. 16-27. Springer: Berlin, 1978.

[2] S. Alpern. Measure preserving homeomorphisms of $\mathbb{R}^{n}$. Indiana University Math. J. 28 (1979), No. $6,957-960$.

[3] S. Alpern. Nonstable ergodic homeomorphisms of $\mathbb{R}^{4}$. To appear.

[4] J. R. Choksi \& S. Kakutani. Residuality of ergodic measurable transformations and of ergodic transformations which preserve an infinite measure. Indiana University Math. J. 28 (1979), 453-469.

[5] N. A. Friedman. Introduction to Ergodic Theory. Von Nostrand Reinhold: New York, 1970.

[6] P. R. Halmos. Approximation theories for measure preserving transformations. Trans. Amer. Math. Soc. 55 (1944), 1-18.

[7] P. R. Halmos. In general a measure preserving transformation is mixing. Ann. of Math. 45 (1944), 786-792.

[8] P. R. Halmos. Lectures on Ergodic Theory. Publ. of Math. Soc. of Japan no. 3. Tokyo, 1956. 